\title{
Evaluation of Fat Tissue Deposition Within the Tongue Via Near- Infrared Interactance
}

\author{
Eriko Ando, PhD1; Yuko Shigeta, DDS, PhD ${ }^{1 *}$; Chieko Ishikawa, PhD¹; Shuji Shigemoto1; Takumi Ogawa, DDS, PhD; Glenn T. \\ Clark, DDS, MS²; Reyes Enciso. $\mathrm{PhD}^{3}$
}

\begin{abstract}
${ }^{1}$ Department of Fixed Prosthodontics, School of Dental Medicine, Tsurumi University, Yokohama, Japan, ${ }^{2}$ Orofacial Pain/Oral Medicine Center, Division of Periodontology, Diagnostic Science \& Dental Hygiene, Herman Ostrow School of Dentistry, University of Southern California, Los Angeles, California, USA, ${ }^{3}$ Division of Dental Public Health and Pediatric Dentistry, Herman Ostrow School of Dentistry, University of Southern California, Los Angeles, California, USA
\end{abstract}

\begin{abstract}
Study Objectives: In this study, the fat deposition within the tongue was evaluated via near-infrared interactance (NIR). The aim of this study was to investigate the feasibility of using NIR spectroscopy for measurement of tongue fat, and the relationship between the tongue fat and OSA status/severity.

Methods: Forty-five patients (34 males, 11 females) who were recruited consecutively had OSA/snoring symptoms and also underwent polysomnography. As control subjects, 28 volunteers (17 males, 11 females) were recruited. The variables of body composition were measured via a body composition meter. The body and tongue fat were evaluated via NIR spectroscopy.

Results: In all subjects (all control patients and all patients with OSA), there was no significant correlation between tongue fat NIR and age. In all males, there was a significant positive correlation between tongue fat NIR and age. The tongue deposition in male patients with OSA was higher than in the male control patients. In all subjects (all control patients, and all patients with OSA), there was significant positive correlation between percent of body fat and tongue fat NIR. There was no significant correlation between tongue fat NIR and AHI in male patients with OSA. There was significant positive correlation in female patients with OSA.

Conclusions: It is suggested that tongue fat deposition may be associated with age, sex, body fat, OSA status (only in males) and AHI (only in females).

Keywords: near-infrared interactance; obstructive sleep apnea; OSA; tongue fat

Citation: Ando E, Shigeta Y, Ishikawa C, Shigemoto S, Ogawa T, Clark GT, Enciso R. Evaluation of fat tissue deposition within the tongue via near-infrared interactance. J Dent Sleep Med. 2019;6(4)
\end{abstract}

\section{INTRODUCTION}

In the pathogenesis of obstructive sleep apnea (OSA), deposition of fat in the upper airway soft tissue has been listed as one of the important factors in previous studies. ${ }^{1,2}$

The excessive fat tissue deposition within the tongue was observed on magnetic resonance imaging (MRI) in the patients with $\mathrm{OSA}^{3},{ }^{3}$ and it was considered that tongue enlargement may occur due to overweight.

In our previous study, ${ }^{4}$ we investigated the influence of the size ratio of the tongue and mandible on the upper airway with three-dimensional reconstructed models from computed tomography (CT) data. Tongue volume increases and airway volume decreases with an increasing body mass index (BMI). These alterations of tongue and airway with increasing weight are a likely factor in the development of OSA.

In previous studies using CT/MRI, it has been suggested that there are sex differences, including differences in distribution of the fat tissue deposition. ${ }^{5}$ However, when using CT/MRI, we must consider the physical/economic burden on the patients. The nearinfrared interactance (NIR) method is used for analyzing body composition. ${ }^{6}, 7$ This method was applied for predicting cardiovascular events, as well as evaluation of obesity. $^{8}$

Therefore, we hypothesized, based on the results of our previous study using CT data, that tongue fat deposition is one of the risk factors of OSA. Moreover, the findings of tongue fat in the CT/MRI data were already reported by several previous studies. However, the potential of NIR spectroscopy for tongue fat have not been investigated. Therefore, in this current study, we assessed the potential of NIR spectroscopy for measurement of tongue fat, and for investigating the relationship between the tongue fat and OSA status/severity.

\section{METHODS}

\section{Subjects}

In this study, 45 patients ( 34 males aged 44 to 81 years, 11 females aged 33 to 73 years) were recruited consecutively from April to December in 2014 who had OSA/snoring symptoms and also underwent polysomnography. As control subjects, 28 volunteers (17 males aged 25 to 69 years, 11 females aged 25 to 58 years) from our university and hospital staff were recruited. The control subjects had no subjective symptoms regarding sleep apnea syndrome, such as snoring or excessive 
daytime sleepiness. All subjects gave written informed consent to participate in this study approved by the Ethical Committee at Tsurumi University Dental Hospital (Approval number: 1126).

In one of the patients with OSA, her body exceeded the measurement limit of the body composition meter. Therefore, for that patient, only NIR spectroscopy data and not body composition data were available.

\section{Measurement of Body Composition}

The variables of body composition were measured via a body composition meter (Karada Scan, OMRON, Kyoto, Japan). Our inquiry points of body composition were as follows: BMI $\left(\mathrm{kg} / \mathrm{m}^{2}\right)$; percentage of body fat $(\%)$. The subjects' height $(\mathrm{cm})$ for calculating BMI was measured with a digital stadiometer. Percentage of body fat was directly measured with bioelectrical impedance analysis using the body composition meter.

\section{Measurement of Body and Tongue Fat}

The arm and tongue fat were evaluated via NIR spectroscopy (FITNESS ANALYZER BFT-3000, Kett Electric Laboratory, Tokyo, Japan). (Figure 1) The measurement sites were the center of the biceps brachii muscle (arm fat NIR) and the tongue dorsal surface (tongue fat NIR). It has been reported that there is significant correlation between the spectra of biceps brachii muscle and percentage of body fat in Japanese subjects. ${ }^{9}$ The surface of the tongue is in a humectation state. Preliminary research described that the NIR spectra obtained by NIR spectroscopy were not affected by the humectation state of the measurement site. ${ }^{10}$ Therefore, NIR spectroscopy has the potential for the quantitative evaluation of tongue fat deposition. During measurement, the measurement site was shaded with a sponge or jig to avoid the influence of environmental light and to obtain a stable measured value.

In measuring body fat, the probe of NIR spectroscopy was positioned perpendicularly on the surface of the brachii muscle according to manufacturer instructions. In measuring tongue fat, heat-shrinkable tubing was used to make the measurement jig. The center of the measuring site was provided at $20 \mathrm{~mm}$ from the tip of tongue via the jig (Figure 2). The multiple time measurement of tongue fat to verify the repeatability of NIR was difficult due to the shape and size of the probe and induction of vomiting reflex. Therefore, the tongue fat was measured two times for each subject, and mean value was used as "tongue fat NIR", to decrease the influence of measurement error.

\section{Statistical Analysis}

Statistical analysis was carried out with SPSS 12.0J
(SPSS Japan Inc. Tokyo, Japan) with a significance level set at $P<0.05$.

First, we analyzed our variables for normality. The linear correlations involving the variables, which failed to pass the Kolmogorov-Smirnov test in all subjects and each subgroup (OSA status: control patients and patients with OSA; males and females), were performed with Spearman test. The other correlations between variables were analyzed with Pearson test. In addition, the distribution of each variable was compared between the subgroups with the $t$ test.

\section{RESULTS}

\section{Distribution of Age, BMI, Percentage of Fat Tissues, and OSA Severity}

Table 1 shows the descriptive statistics for age, BMI, percentage of fat tissues, and OSA severity (AHI, only in patients with OSA).

There was a significant difference in age and BMI between all control patients and those with OSA $(t$ test; age: $P<.001$, BMI: $P=.011)$. However, male control patients and male patients with OSA were matched for BMI, and female control patients and female patients with OSA were also matched for BMI ( $t$ test; male group: $P=.177$, female group: $P=.084$ ).

There was no significant difference in AHI between male and female patients with OSA ( $t$ test: $P=.337)$.

Figure 1. Near Infrared Interactance spectroscopy.

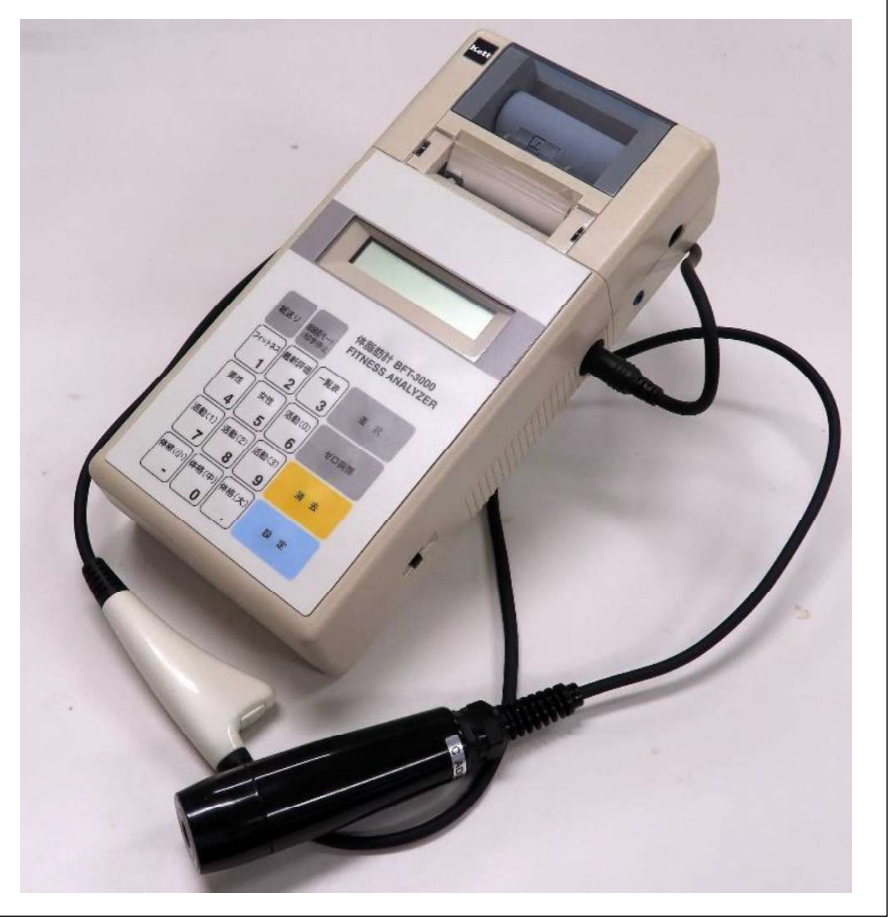


Figure 2. Fabrication of jig for measuring of tongue fat.

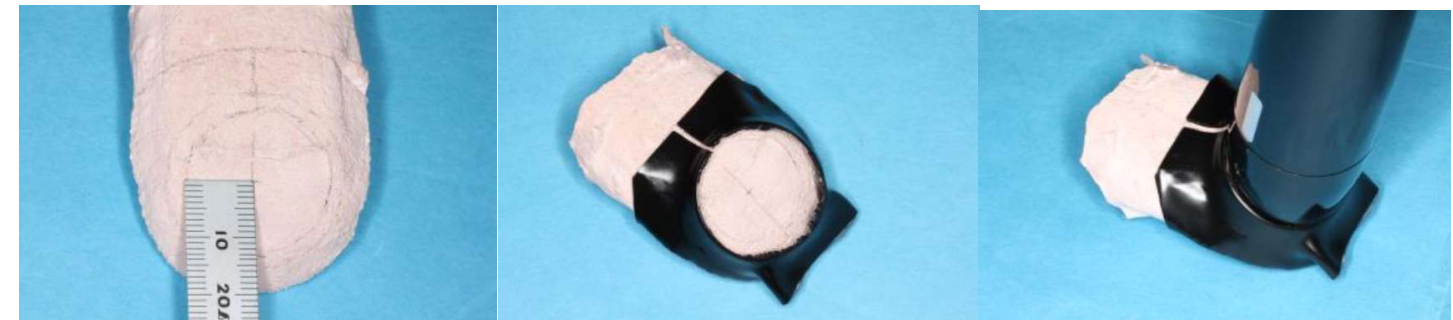

Figure 3. Simple correlation between percent of body fat and arm fat NIR in all subjects.

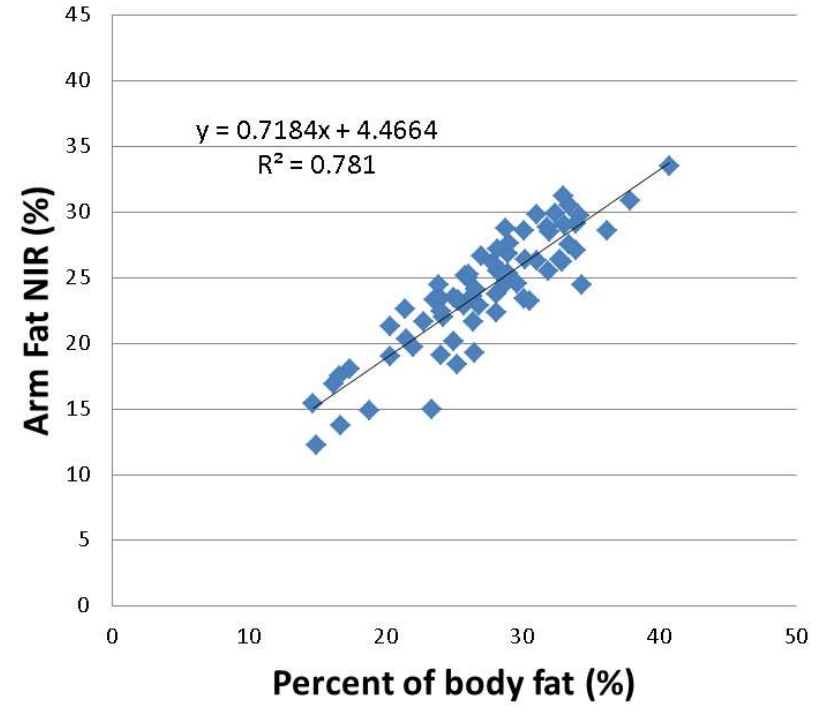

Percentage of body fat in all females was significantly greater than in all males, though male subjects had high BMI ( $t$ test: percentage of body fat: $P<.001$. There was no significant different in tongue fat NIR between all control patients and all patients with OSA $(t$ test: $P=.127)$

\section{Relationship Between Percentage of Body Fat and Arm Fat NIR}

In all patients, there was a highly significant positive correlation between percentage of body fat and arm fat NIR (Pearson analysis $\mathrm{r}=.884, P<.001$ ) (Figure 3 ).

There was a highly significant positive correlation between percentage of body fat and arm fat NIR in all control patients (Pearson analysis $\mathrm{r}=.947, P<.001$ ) and in all patients with OSA (Pearson analysis $\mathrm{r}=.882, P$ $<.001)$.

\section{Relationship Between Percentage of Body Fat and BMI}

In all subjects, there was significant positive correlation between percent of body fat and BMI (Pearson analysis $\mathrm{r}=.490, P<.001)$

There was no significant correlation between percentage of body fat and BMI in all control patients (Spearman analysis $\mathrm{r}=.171, P=.384$ ). However, there was a significant positive correlation between percentage of body fat and BMI in all patients with OSA (Pearson analysis $\mathrm{r}=.539, P<.001)$. When all patients with OSAs were grouped according to sex, there was significant positive correlation between percentage of body fat and $\mathrm{BMI}$ in male patients (Pearson analysis $\mathrm{r}=.748, P<.001$ ), and in female patients (Spearman analysis $\mathrm{r}=.698, P$ $=.025)$.

\section{Tongue Fat Deposition}

In all subjects, there was no significant difference in the tongue fat NIR between all control patients and all patients with OSA ( $t$ test; $P=.127$ ). There was a significant difference in the tongue fat deposition between male control patients and males with OSA ( $t$ test; $P=.027$ ). However, in the female group, there was no significant difference in the tongue fat deposition between control patients and patients with OSA ( $t$ test; $\mathrm{p}=.091)$.

\section{Relationship Between Tongue Fat NIR and Age}

In all subjects, all control patients, and all patients with OSA, there was no significant correlation between tongue fat NIR and age (Spearman analysis, all subjects: $\mathrm{r}$ $=.192, P=.104$, all control patients: $\mathrm{r}=.231, P=.237$, all patients with OSA: $r=-.000, P=.999$ ).

In the male group, there was a significant positive correlation between tongue fat NIR and age (Spearman analysis, $\mathrm{r}=.372, P=.007)$. However, in the female group, there was no significant correlation (Pearson analysis, $r$ $=.096, P=.671)$.

\section{Relationship Between Percent of Body Fat and Tongue Fat NIR}

In all subjects, all control patients, and all patients with OSA, there was significant positive correlation between percent of body fat and tongue fat NIR (Spearman analysis: all subjects: $\mathrm{r}=.626, P<.001$, all control patients: $\mathrm{r}=.607, P=.001$, all patients with OSA: $\mathrm{r}=.639, P$ 
Table 1. Descriptive statistics for age, body mass index, percentage of fat tissues, and obstructive sleep apnea severity (apnea-hypopnea index).

\begin{tabular}{|c|c|c|c|c|c|}
\hline \multirow[t]{2}{*}{ Our variables } & & \multicolumn{3}{|c|}{ Min.-Max. (Mean \pm SD) } & \multirow[b]{2}{*}{ M-F } \\
\hline & Group & All & Male & Female & \\
\hline \multirow{3}{*}{$\begin{array}{l}\text { Age (year- } \\
\text { old) }\end{array}$} & Controls & $25-69(41 \pm 11.2)$ & $25-69(40 \pm 11.3)$ & $25-58(43 \pm 11.2)$ & NS \\
\hline & OSAs & $37-81(61 \pm 12.1)$ & $44-81(62 \pm 12.4)$ & $37-73(57 \pm 10.4)$ & NS \\
\hline & Con-OSA & $* *$ & $* *$ & $* *$ & \\
\hline \multirow[t]{3}{*}{ BMI $\left(\mathrm{kg} / \mathrm{m}^{2}\right)$} & Controls & $19.1-33.2(23.9 \pm 3.6)$ & $19.2-33.2(25.3 \pm 3.8)$ & $19.1-26.0(21.8 \pm 1.9)$ & $* *$ \\
\hline & OSAs & $19.3-35.2(26.3 \pm 4.1)$ & $20.4-35.2(26.9 \pm 4.1)$ & $19.3-33.6(24.3 \pm 3.8)$ & NS \\
\hline & Con-OSA & * & NS & NS & \\
\hline \multirow{3}{*}{$\begin{array}{l}\text { Percent of } \\
\text { body fat }(\%)\end{array}$} & Controls & $14.7-33.0(25.5 \pm 5.11)$ & $14.7-31.0(22.9 \pm 4.75)$ & $26.1-33.0(29.6 \pm 2.15)$ & $* *$ \\
\hline & OSAs & $14.9-40.7(28.2 \pm 5.44)$ & $14.9-34.3(26.9 \pm 4.72)$ & $22.0-40.7(32.7 \pm 5.61)$ & * \\
\hline & Con-OSA & * & $* *$ & NS & \\
\hline \multirow{3}{*}{$\begin{array}{l}\text { Arm fat NIR } \\
(\%)\end{array}$} & Controls & $13.7-31.2(23.9 \pm 4.39)$ & $13.7-26.3(21.4 \pm 3.59)$ & $25.2-31.2(27.7 \pm 2.10)$ & $* *$ \\
\hline & OSAs & $12.2-39.2(24.4 \pm 4.98)$ & $12.2-30.5(23.0 \pm 4.13)$ & $19.7-39.2(28.8 \pm 5.03)$ & $* *$ \\
\hline & Con-OSA & $\mathbf{N S}$ & NS & $\mathbf{N S}$ & \\
\hline \multirow{3}{*}{$\begin{array}{ll}\text { Tongue } & \text { fat } \\
\text { NIR }(\%) & \end{array}$} & Controls & $8.8-22.4(16.0 \pm 3.68)$ & $8.8-20.9(14.1 \pm 2.93)$ & $13.1-22.4(18.9 \pm 2.75)$ & $* *$ \\
\hline & OSAs & $10.6-27.9(17.4 \pm 3.75)$ & $10.6-22.5(16.1 \pm 2.80)$ & $15.0-27.9(21.3 \pm 3.62)$ & $* *$ \\
\hline & Con-OSA & NS & $*$ & NS & \\
\hline $\begin{array}{l}\text { AHI } \\
\text { (events/h) }\end{array}$ & OSAs & $7.6-101.0(37.4 \pm 20.32)$ & $15.4-87.6(39.4 \pm 18.04)$ & $7.6-101.0(31.0 \pm 26.15)$ & NS \\
\hline
\end{tabular}

$<.001)$.

\section{Relationship Between Tongue Fat NIR and OSA Severity (AHI)}

In all patients with OSA, there was no significant correlation between tongue fat NIR and AHI (Spearman analysis: $\mathrm{r}=-.012, P=.939$ ).

There was no significant correlation between tongue fat NIR and AHI in male patients with OSA (Spearman analysis $\mathrm{r}=.048, P=.788$ ), however there was a significant positive correlation in female patients with OSA (Pearson analysis: $\mathrm{r}=.763, P=.006$ ).

\section{DISCUSSION}

\section{The Validity of the Experimental Method}

In all patients and in each subgroup, there was a highly significant positive correlation between percentage of body fat and arm fat NIR.

Obesity is generally evaluated using BMI, waist circumference, waist-to-hip ratio, and skinfold thickness. ${ }^{11-}$ 13 However, these methods have no potential for distinguishing between lean body mass and fat mass. ${ }^{14}$ To investigate the fat mass, there is bioelectrical impedance analysis ${ }^{15}$ and several other accurate methods ${ }^{16-18}$ such as MRI, CT, and dual-energy x-ray absorptiometry. However, it is difficult to apply these methods for large-scale population-based surveys. There is the radiation exposure issue when CT is used. In addition, the impedance analysis method is susceptible to moisture such as that derived from sweat. An NIR analyzer measures the percentage of body fat using the light absorption differences depending on tissue composition. ${ }^{19}$ Therefore, in this study, the NIR method was applied to evaluate tongue fat deposition.

However, the NIR spectroscopy has been not applied to measure tongue fat deposition. Therefore, the potential of NIR method for measurement of tongue fat has to be assessed with a control device.

In this study, the relationship between percentage of body fat via the body composition meter and percentage of arm fat via NIR spectroscopy was investigated. There was significant positive correlation between percentage of body fat and arm fat NIR. This result proves the potential of using NIR spectroscopy as a substitute for a body composition analyzer.

The measurement site of the tongue was determined at $20 \mathrm{~mm}$ from the tip of the tongue to prevent subject discomfort related to size of the probe and the overall procedure. In addition, Miller et al. ${ }^{20}$ investigated the composition and organization of tongue in the adults and found the greater adipose in the anterior region of tongue. Therefore, in this current study, the percentage of tongue fat was measured at the anterior region on the tongue dorsal surface. 


\section{Relationship Between Percentage of Body Fat and BMI}

In all subjects, there was significant positive correlation between percentage of body fat and BMI. There was no significant correlation between percentage of body fat and BMI in all control patients. However, there was a significant positive correlation between percentage of body fat and BMI in all patients with OSA.

Blaak $^{21}$ investigated the sex differences in fat metabolism. The percentage body fat in women is generally higher than that in men. Fat tissue is deposited on the gluteal-femoral region in women, and on the visceral depot in men. In our previous study ${ }^{22}$ we investigated the sex differences of fat distribution in the neck region and its influence on the airway. In males, there was significantly more internal fat (defined as total fat minus subcutaneous fat) than in females. From the results of previous studies, it was suggested that there are sex differences in fat metabolism and deposition.

However, in the patients with OSA, there were no sex differences regarding the relationship between percentage of body fat and BMI within the limitations of this study.

\section{Tongue Fat Deposition}

In all subjects, there was no significant difference in the tongue fat NIR between all control patients and all patients with OSA. On one hand, in the male group, there was a significant difference in the tongue fat between male control patients and male patients with OSA. On the other hand, in the female group, there was no significant difference in the tongue fat due to OSA status.

In 2004, Kim et al. ${ }^{23}$ investigated the tongue fat deposition using the MRI data. They reported that the mean tongue fat percentage in the upper mid retropalatal region was $15.2 \%$ in the control patients, and $17.4 \%$ in the patients with apnea. In our study, we obtained very similar results: the mean tongue fat NIR was $16.0 \%$ in the control group and $17.4 \%$ in the patients with OSA. According to Kim et al., ${ }^{23}$ there was no significant difference in tongue fat between control patients and the OSA group; we did find a significant difference only in males. According to the data from Kim et al., there was significant difference in the tongue fat percentage before matching for age, BMI, sex, and race. Our subjects were not matched for age and sex, though they were matched for BMI according to sex. In addition, it is suggested that the tongue fat deposition was associated with age in the male group. From these findings, it was suggested that age and sex might be important contributing factors to tongue fat deposition in addition to BMI.

In addition, despite matching for BMI between control patients and those with OSA for both sexes, percentage of body fat in the male patients with OSA was significantly greater than in the male control patients. This result suggests that male patients with OSA have a specific fat tissue deposition. Therefore, it was considered that the greater fat deposition of the tongue was one of the specific fat tissue depositions in the male patients with OSA.

If our results hold in future research confirmatory studies, NIR has the potential to measure the tongue fat.

\section{Relationship Between Tongue Fat NIR and Age}

In all subjects (all control patients, and all patients with OSA), there was no significant correlation between tongue fat NIR and age. However, in the male patient group, there was a significant positive correlation between tongue fat NIR and age.

In 1987, Bässler et al. ${ }^{24}$ histologically examined the 170 autopsy tongues and investigated the process of the development of age-related atrophy. The authors reported that the development of age-related atrophy in the tongue was characterized by musculature with progressive lipomatosis. In this study, there was significant positive correlation between tongue fat NIR and age in the male patients with OSA. Our findings partially corresponded with their results.

In 2012, Butler et al. $^{25}$ examined the tissue composition of the tongue to identify risk factors for aspiration in the elderly. They concluded that the decrease of isometric strength was associated with greater adiposity in the posterior tongue. It was considered that the fat tissue deposition in the tongue due to aging, obesity, and muscular hypotonia may be a risk factor for the development and worsening of OSA. In the current study, the tongue fat in the male patients with OSA was greater than that in the male control patients, and significantly increased with aging. In 1999, Mortimore et al. ${ }^{26]}$ investigated the sex differences and aging changes in tongue protrusion strength. They concluded that tongue protrusion is greater in males compared to females, and decreases with age.

From our results, it was suggested that the age-related atrophy of the tongue leads to OSA in males, who have a specific fat deposition.

\section{Relationship Between Percentage of Body Fat and Tongue Fat NIR}

In all subjects, there was significant positive correlation between percentage of body fat and tongue fat NIR.

In 2009, Bruno et al. ${ }^{27}$ evaluated the body composition in patients with OSA compared to control patients. From the result of the tissue composition measurement in the oral region via dual-energy $\mathrm{x}$-ray absorptiometry, it was revealed that the fat-free mass in patients with OSA was significantly less than in the control group.

From the results of previous studies, it has been suggested that tongue fat influences deglutition and 
respiratory functions. Furthermore, in 2002, Welch et al. ${ }^{28}$ described that the upper airway caliber increase due to decreasing the size of the parapharyngeal fat pads and lateral pharyngeal wall with weight loss. In this study, a significant correlation between tongue fat increase and increasing body fat has been found. In a future study, the possible decrease of tongue fat due to weight loss should/will be investigated.

\section{Relationship Between Tongue Fat NIR and OSA Severity (AHI)}

There was no significant correlation between tongue fat NIR and AHI in male patients with OSA. However, there was a significant positive correlation in female patients with OSA.

The tongue fat increased with increasing percentage of body fat, and in the male patients with OSA it was significantly greater than that in the male control patients. In addition, there was a significant positive correlation between percentage of body fat and BMI in all patients with OSA. In our previous study ${ }^{4}$ it was revealed that the tongue volume increased with increasing BMI. From these findings, it was suggested that the increasing tongue fat volume occurs due to fat deposition in patients with OSA. Therefore, it was considered that the greater fat deposition of the tongue was one of the specific fat tissue depositions in male patients with OSA, and the tongue fat deposition is one of the risk factors for the development of OSA in males.

However, in the female patients with OSA, there was a significant correlation between tongue fat and OSA severity, and there was no significant difference in tongue fat due to OSA status. Obesity has long been known to be associated with OSA, and in both sexes BMI correlates positively with the severity of the disease..$^{28,29}$ In the current study, the tongue fat increased with increasing percentage of body fat. Nevertheless, in the male patients with OSA, there was no significant correlation between tongue fat and OSA severity. There is a possibility that sex differences of the fat distribution in the tongue have different implications for men and women. Although fat accumulates in the tongue with weight gain, ${ }^{30}$ women in general have lower Mallampati scores, suggesting that fat does not play as large a role in the female tongue as it does in the male tongue. ${ }^{31}$ Our results corresponded with previous studies.

The average tongue fat NIR in the females was higher than in the males, more than $5 \%(t$ test: $P<.001)$.

In adults in general, approximately $3 \%$ to $7 \%$ of males and $2 \%$ to $5 \%$ of females have OSA associated with accompanying daytime sleepiness. ${ }^{32,}{ }^{33}$ However, female patients with OSA are apt to be underdiagnosed due to lifestyle and sociocultural factors, in addition to having different clinical symptoms of OSA. ${ }^{34}$

In 2001, Kapsimalis and Kryger ${ }^{35}$ reviewed the sex differences associated with the risk factor of OSA, and suggested that the decrease of sex-related protective effect occurs as a result of menopause. The genioglossus activity is lower in postmenopausal women and the female hormones (possibly progesterone) have a substantial effect on upper airway dilator muscle activity. ${ }^{36}$ Most of the female patients with OSA in our study ( 8 of 11) were older than 50 years, which is the median age of menopause in Japanese women. ${ }^{37}$ The hypoactivity in the extrinsic muscles of the tongue may induce a fatty metamorphosis. In addition, there is a report that investigated the histopathologic changes in the base of the tongue of rats by using an experimental menopause model. ${ }^{38}$ It was suggested that menopause induces the significant histopathologic changes in the base of the tongue, similar to that in patients with OSA, regarding lymphocyte infiltration, polymorphonuclear leukocyte infiltration, and subepithelial edema.

Our results may indicate a contributing risk factor of OSA for females, in particular after menopause. Additional confirmatory studies are necessary.

\section{CONCLUSION}

In this study, the fat deposition within the tongue was evaluated via NIR to reveal the characteristics of tongue fat deposition and its relationship with age, sex, body composition, and AHI.

From our results, it was suggested that tongue fat deposition may be associated with age, sex, body fat, OSA status (only in males) and AHI (only in females).

\section{ACKNOWLEDGMENTS}

Dr. Shigeta was partially supported by MEXT grant \# 24500560 .

\section{REFERENCES}

1. Shelton KE, Woodson H, Gay S, Suratt PM. Pharyngeal fat in obstructive sleep apnea. Am Rev Respir Dis.1993;148(2):462-466.

2. Schwab RJ, Gupta KB, Gefter WB, Metzger LJ, Hoffman EA, Pack AI. Upper airway and soft tissue anatomy in normal subjects and patients with sleep-disordered breathing: significance of the lateral pharyngeal walls. Am J Respir Crit Care Med. 1995;152(5 Pt 1):16731689 .

3. Horner RL, Mohiaddin RH, Lowell DG, et al. Sites and sizes of fat deposits around the pharynx in obese patients with obstructive sleep apnoea and weight matched controls. Eur Respir J. 1989;2(7):613622.

4. Shigeta Y, Ogawa T, Ando E, Clark GT, Enciso R. Influence of tongue/mandible volume ratio on oropharyngeal airway in Japanese male patients with obstructive sleep apnea. Oral Surg Oral Med Oral Pathol Oral Radiol Endod. 2011;111(2):239-243.

5. Shigeta Y, Enciso R, Ogawa T, Ikawa T, Clark GT. Cervical CT derived neck fat tissue distribution differences in Japanese males and females and its effect on retroglossal and retropalatal airway volume. Oral Surg Oral Med Oral Pathol Oral Radiol Endod. 2008;106(2):275-284.

6. Conway JM, Norris KH, Bodwell CE. A new approach for the 
estimation of body composition: infrared interactance. Am $\mathrm{J}$ Clin Nutr. 1984;40(6):1123-1130.

Conway JM, Norris KH. Non-invasive body composition in humans by near infrared interactance. Ellis KJ. Yasumura S, Morgan WD. In vivo body composition studies, Ed., proceedings of international symposium held at Brookhaven National Laboratory, New York published by the Institute of Physical Sciences in Medicine, London, 163-170.

8. Pajunen P, Jousilahti P, Borodulin K, Harald K, Tuomilehto J, Salomaa V. Body fat measured by a near-infrared interactance device as a predictor of cardiovascular events: the FINRISK'92 cohort. Obesity (Silver Spring). 2011;19(4):848-852.

Sawai S, Shirayama M, Mutoh Y, Miyashita M. Estimation of body fat by near infrared spectroscopic technique. The Japanese Journal of Physical Fitness and Sports Medicine. 1990;39:155-163. [Japanese]

Katsuno K, Fukuyama Y, Nishiyama K, Urata H, Otsuka K. Practical evaluation of the instrument for estimation of body composition by near infrared spectroscopy. Bull Sch Allied Med Sci Nagasaki Univ. 1991;5:23-32. [Japanese]

Lakka HM, Lakka TA, Tuomilehto J, Salonen JT. Abdominal obesity is associated with increased risk of acute coronary events in men. Eur Heart J. 2002;23(9):706-713.

Molarius A, Seidell JC, Sans S, Tuomilehto J, Kuulasmaa K. Waist and hip circumferences, and waist-hip ratio in 19 populations of the WHO MONICA Project. Int $J$ Obes Relat Metab Disord. 1999;23(2):116-125.

Lahti-Koski M. Body Mass Index and Obesity Among Adults in Finland. Academic dissertation, 2001.

Wellens RI, Roche AF, Khamis HJ, Jackson AS, Pollock ML, Siervogel RM. Relationships between the body mass index and body composition. Obes Res. 1996;4(1):35-44. in large epidemiological studies? Nutr J. 2008;7:26.

Goodpaster BH. Measuring body fat distribution and content in humans. Curr Opin Clin Nutr Metab Care. 2002;5(5):481-487.

Glickman SG, Marn CS, Supiano MA, Dengel DR. Validity and reliability of dual-energy X-ray absorptiometry for the assessment of abdominal adiposity. $J$ Appl Physiol. 2004;97(2):509-514.

8hen W, Punyanitya M, Wang Z, et al. Visceral adipose tissue: relations between single-slice areas and total volume. Am J Clin Nutr. 2004;80(2):271-278.

Schreiner PJ, Pitkäniemi J, Pekkanen J, Salomaa VV. Reliability of near-infrared interactance body fat assessment relative to standard anthropometric techniques. J Clin Epidemiol. 1995;48(11):13611367.

Miller JL, Watkin KL, Chen MF. Muscle, adipose, and connective tissue variations in intrinsic musculature of the adult human tongue. $J$ Speech Lang Hear Res. 2002;45(1):51-65.

Blaak E. Gender differences in fat metabolism. Curr Opin Clin Nutr Metabc Care. 2001;4(6):499-502.

Shigeta Y, Enciso R, Ogawa T, Ikawa T, Glenn T. Clark. Cervical CT derived neck fat tissue distribution differences in Japanese males and females and its effect on retroglossal and retropalatal airway volume. Oral Surg Oral Med Oral Pathol Oral Radiol Endod. 2008;106(2):275-284

Kim AM, Keenan BT, Jackson N, et al. Tongue fat its relationship to obstructive sleep apnea. Sleep. 2014;37910):1639-1648.

Bässler R. Histopathology of different types of atrophy of the human tongue. Pathol Res Pract. 1987;182(1):87-97. 1604.
Dehghan M, Merchant AT. Is bioelectrical impedance accurate for use

Butler SG, Lintzenich CR, Leng X, et al. Tongue adiposity and strength in healthy older adults. Laryngoscope. 2012;122(7):1600- force and fatiguability in male and female subjects. Eur Respir J. 1999;14(1):191-195.

27. Bruno E, Alessandrini M, Napolitano B, Padova AD, Daniele ND, Lorenzo AD. Dual-energy X-ray absorptiometry analysis of body composition in patients affected by OSAS. Eur Arch Otorhinolaryngol. 2009;266(8):1285-1290.

28. Welch KC, Foster GD, Ritter CT, et al. A novel volumetric magnetic resonance imaging paradigm to study upper airway anatomy. Sleep. $2002 ; 25(5): 532-542$

29. Thurnheer R, Wraith PK, Douglas NJ. Influence of age and gender on upper airway resistance in NREM and REM sleep. $J$ Appl Physiol. 2001;90(3):981-988.

30. Nashi N, Kang S, Barkdull G, Lucas J, Davidson TM. Lingual Fat at Autopsy. Laryngoscope. 2007;117(8):1467-1473.

31. Davidson T, Patel M. Waist, circumference and sleep disordered breathing. Laryngoscope. 2008;118(2):339-347.

32. Punjabi NM. The Epidemiology of Adult Obstructive Sleep Apnea. Proc Am Thorac Soc. 2008;5(2):136-143.

33. Mirrakhimov AE, Sooronbaev T, Mirrakhimov EM. Prevalence of obstructive sleep apnea in Asian adults: a systematic review of the literature. BMC Pulm Med. 2013;13:10.

34. Quintana-Gallego E, Carmona-Bernal C, Capote F, et al. Gender differences in obstructive sleep apnea syndrome: a clinical study of 1166 patients. Respir Med. 2004;98(10):984-989.

35. Kapsimalis F, Kryger MH. Gender and obstructive sleep apnea syndrome, Part 2: Mechanisms. Sleep. 2002;25(5):499-506.

36. Popovic RM, White DP. Upper airway muscle activity in normal women: influence of hormonal status. $J$ Appl Physiol. 1998;84(3):1055-1062.

37. Tamada T, Iwasaki H. Age at natural menopause in Japanese women. Nihon Sanka Fujinka Gakkai Zasshi. 1995;47(9):947-952. [Japanese]

38. Deveci I, Sürmeli M, Senem Deveci H, et al. Effects of polycystic ovary syndrome and menopause on rat soft palate and base of tongue. Otolaryngol Head Neck Surg. 2013;148(4):595-601.

\section{SUBMISSION \& CORRESPONDENCE INFORMATION}

\section{Submitted for publication November 1, 2018 Submitted in final revised form April 18, 2019 Accepted for publication June 18, 2019}

Address correspondence to: Yuko Shigeta DDS, PhD, Department of Fixed Prosthodontic, School of Dental Medicine, Tsurumi University, 2-1-3 Tsurumi, Tsurumiku, Yokohama, Japan; Email: shigeta-y@tsurumi-u.ac.jp; Phone: +81-45-580-8417; Fax: +81-45-573-9599

\section{DISCLOSURE STATEMENT}

The authors have no conflicts of interest to disclose. 